Article

\title{
Spatial Distribution and Influencing Factors of Chinese Time-Honored Catering Brands in the Five Northwestern Provinces
}

\author{
Haiyan Zhang, Meiping Sun *, Hao Zhang, Lei Zhang and Zhilan Wang \\ College of Geography and Environmental Science, Northwest Normal University, Lanzhou 730070, China \\ 2020212674@nwnu.edu.cn (H.Z.); zhanghao1996n@163.com (H.Z.); 2019212353@nwnu.edu.cn (L.Z.); \\ 2019212357@nwnu.edu.cn (Z.W.) \\ * Correspondence: sunmeiping1982@nwnu.edu.cn
}

check for

updates

Citation: Zhang, H.; Sun, M.; Zhang, H.; Zhang, L.; Wang, Z. Spatial Distribution and Influencing Factors of Chinese Time-Honored Catering Brands in the Five Northwestern Provinces. Sustainability 2021, 13, 3755. https://doi.org/10.3390/ su13073755

Academic Editors: Pablo Marti and Leticia Serrano-Estrada

Received: 3 February 2021

Accepted: 24 March 2021

Published: 28 March 2021

Publisher's Note: MDPI stays neutral with regard to jurisdictional claims in published maps and institutional affiliations.

Copyright: (c) 2021 by the authors. Licensee MDPI, Basel, Switzerland. This article is an open access article distributed under the terms and conditions of the Creative Commons Attribution (CC BY) license (https:// creativecommons.org/licenses/by/ $4.0 /)$.

\begin{abstract}
The Chinese Time-honored Catering Brands (CTCBs) in the five northwestern provinces are the accumulation of culture in the northwest of China and have precious value. Their formation and development process have their own characteristics and rules. In order to clarify the spatial distribution characteristics and influencing factors of CTCBs in these provinces, this study uses geolocated social media data (the data of the CTCBs' directory, location, and per capita consumption level), and applies statistical analysis, kernel density analysis, standard deviational ellipse, and buffer analysis to analyze the spatial distribution characteristics and influencing factors of CTCBs in the five northwestern provinces. The results show that: (1) the spatial development intensity of CTCBs is relatively strong in their original place, and their spatial distribution is uneven. The overall distribution is in the northwest-southeast direction, with strong agglomeration, and tends to be scattered in the periphery of the agglomeration area. There are some areas with higher concentrations distributed in the form of patches. (2) The number and spatial distribution of old brands with different grades and tastes have different characteristics. (3) The spatial distribution pattern of CTCBs is formed under a variety of factors, such as historical and traditional culture, regional traffic conditions and urban development patterns, etc.
\end{abstract}

Keywords: the five northwestern provinces; Chinese Time-honored Catering Brands; spatial distribution; influence factor

\section{Introduction}

The Chinese Time-honored Brands (CTBs), which have been baptized for many years, are the epitome of people's life and production process, carve the mark of the boom and decline of the city, and have precious value [1]. In April 2006, the Ministry of Commerce of the People's Republic of China issued the "Standards for the Recognition of Chinese Timehonored Brands (Trial)" and the "Revitalization of Time-honored Brands" program, which defined CTBs as brands with products, skills, or services passed down from generation to generation, distinctive traditional Chinese cultural characteristics and profound cultural heritage, and that are widely recognized by society [2]. The recognition conditions of CTBs are that they are in good operating condition, have a reputation which is recognized by society, and have a strong ability of sustainable development, etc. According to these conditions, the Ministry of Commerce has recognized two batches of CTBs since 2006, with a total of 1128 brands [3]. Among all the CTBs, Chinese Time-honored Catering Brands (CTCBs) have the largest number and proportion. As the most important part of the CTBs, CTCBs are rich in types and have their own characteristics [4]. According to the region, CTCBs can be divided into different tastes: Shandong taste, Sichuan taste, Guangdong taste, Jiangsu taste, Fujian taste, Zhejiang taste, Hunan taste, and Anhui taste $[5,6]$. Due to differences in ingredients, time spent, cooking methods, etc., the per 
capita consumption of CTCBs is also different, so CTCBs can be divided into different grades (high-consumption grade, medium-consumption grade, and low-consumption grade) [7]. The types, grades, and quantities of CTCBs in a region are the result of the interaction of space, time, and human factors [8], so it is of great significance to analyze the spatial distribution characteristics of CTCBs and their influencing factors.

At present, foreign studies on time-honored restaurants are mainly from the perspectives of time-honored brands marketing, brand development experiences, and consumers' purchase intention $[9,10]$. French and German wine are typical representations. The status of wine in France and Germany is equivalent to that of Chinese time-honored restaurants in China, and its complete industrial chain and sales methods have important reference significance for the development of Chinese time-honored restaurants. For example, Ugaglia et al. gave a very comprehensive introduction to the French wine industry, including the French wine industry's state and current position in the wine world, the sector of grape-growing and winemaking in France and the distribution channels, the strengths and weaknesses of wine industry development [11]. Dressler et al. have deployed an ethnographic observation of wineries' websites and social media interaction delivering a typology of winery small and medium-sized enterprises's branding strategies. They thought that sustainability needed to be addressed by all brand strategies [12]. Dressler and Paunovic pointed out that the wine industry, as a part of the food industry, needed to establish a consistent, strategically founded product communication [13]. Domestic researches on time-honored catering brands mainly contain strengths and weaknesses of CTCBs, their current situation, development and protection, and revitalization measures [14]; studies from the fields of management $[15,16]$, history and architecture, containing brand's management and innovation, sustainable development and the protection spatial planning and layout of intangible cultural heritage [17-19]; temporal and spatial distribution of long-established brands and influencing factors in a certain city or country [20,21], etc. For example, Guo et al. combined the current trend of experiencing an economic environment, and used the theory of emotional design to analyze the remodeling of the image of Wuhan's time-honored restaurant brands, and put forward some suggestions for remolding the time-honored restaurant brands from three aspects [22]. Mu constructed purchasing model of the timehonored brands and found that consumers' nostalgic psychology had a positive effect on consumer's perception and buying behavior [23]. Zhang analyzed the micro-marketing status of 117 time-honored enterprises in Beijing, summarized their existing problems, and gave some corresponding micro-marketing strategies [24]. A few scholars combine time-honored brands with their geographical space for research $[25,26]$. Gu et al. found that the spatial distribution of Nanjing's catering industry mainly presented a pattern of symbiosis development which took Xinjiekou as the service core [19]. Zhang et al. found that restaurants in the main urban area of Beijing showed a decreasing distribution pattern from the city center to the periphery. Restaurants with different per capita consumption levels and online word-of-mouth levels showed a spindle-shaped distribution characteristic of "more in the middle and less at both ends" [27].

However, there are not many studies on the distribution and influencing factors of CTCBs in the mesoscale space, especially in Northwest China. Therefore, this study analyzes the spatial distribution characteristics and their influencing elements of the timehonored catering brands in five northwestern provinces and then analyzes the spatial distribution of them from two aspects: grades and tastes. It fills in the blank of the discussion of the time-honored catering brand's spatial pattern differences and grasps the development status and changing trend of the time-honored brands in northwest China, which promotes the sustainable development of the catering industry and has certain reference significance for optimizing the pattern of time-honored catering outlets in cities.

\section{Study Area}

As important economic belts linking China's central and eastern regions, the five provinces, Shaanxi, Gansu, Ningxia, Xinjiang, and Qinghai, located in the northwest of 
China, connect Central and West Asia with Europe, building new bridges between Asia and Europe and radiating to Central Asia. It is a gathering place for ethnic minorities, with a wide range of cuisines, and its food style is more unique, natural, and substantial than other areas. For example, Lanzhou Beef Noodles are popular, Highland barley wine, Mutton, and Bread Pieces in Soup have unique charms, and Xifeng Liquor is famous all over the country. They are of great significance for Chinese cuisine to go global. Therefore, we take the five provinces as the study area, and mainly analyze the spatial distribution characteristics and influencing factors of the existing Chinese traditional catering brands recognized by the Ministry of Commerce in these five provinces.

\section{Materials and Methods}

\subsection{Data}

The data of the CTCBs' directory were obtained from the website of the China Timehonored Brand Information Management (http:/ /zhlzh.mofcom.gov.cn/, accessed on 23 November 2020). The location information came from the Baidu map geo-coordinate picker tool (http:/ / api.map.baidu.com/lbsapi/getpoint/, accessed on 30 November 2020) and we looked up price data for these brands from the website Dianping.com.

\subsection{Methods}

\subsubsection{Location Entropy}

Location entropy mainly analyzes the spatial distribution and concentration of a certain element. The larger the value is, the higher the development intensity of this business in the region is. It can be calculated as follows [28]:

$$
L Q_{i j}=\frac{q_{i j} / q_{j}}{q_{i} / q}
$$

where $L Q_{i j}$ is the location entropy of time-honored catering outlets in the area of $j, q_{i j}$ and $q_{i}$ are the numbers of time-honored outlets originating in Province $C$ distributed in the country and local respectively. $q_{j}$ and $q$ are the number of all time-honored brands distributed in the province of $j$ and the whole country respectively. If $L Q_{i j}>1$, the development intensity of the time-honored catering business in the province of $j$ is higher than the average level of other provinces and vice versa. If $L Q_{i j}>1.5$, the development intensity of the business in the province of $j$ has a significant advantage in this province.

\subsubsection{Average Nearest Neighbor Analysis}

We obtain the nearest neighbor index by calculating the average distance between every two points and its nearest point to describe the spatial distribution pattern of the time-honored brands. The index is defined by [29]:

$$
R=\text { dobs } / \operatorname{dexp}
$$

where dobs is the actual distance between the point and its closest point and $\operatorname{dexp}$ is the expected closest distance. If the points are randomly distributed in space, then $R=1$; if the points are in agglomeration distribution, $R<1$; if the points are uniformly distributed, $R>1$.

\subsubsection{Density Kernel Analysis}

Density kernel analysis is a method to re-establish the probability density function from random sampling points by calculating the density of surrounding points. The calculation principle is to give different weights to each point falling into a specific search area (the radius of the area is $h$ ). With the increase of the distance from the center of the specific search area, the weights of the points decrease, and the weight of the point at the maximum distance is 0 . The calculated weights are distributed smoothly [30]. 


\subsubsection{Standard Deviational Ellipse}

Standard deviation ellipse is a common method to quantitatively analyze and describe the spatial distribution characteristics of research objects through three basic parameters: azimuth angle, long axis, and short axis [31]. The azimuth angle can be calculated by:

$$
\begin{aligned}
S D E_{x} & =\sqrt{\frac{\sum_{i=1}^{n}\left(x_{i}-\bar{X}\right)^{2}}{n}} \\
S D E y & =\sqrt{\frac{\sum_{i=1}^{n}\left(y_{i}-\bar{Y}\right)^{2}}{n}} \\
A=\left(\sum_{i=1}^{n} \widetilde{x}_{i}^{2}-\sum_{i=1}^{n} \widetilde{y}_{i}^{2}\right), B & =\sqrt{\left(\sum_{i=1}^{n} \widetilde{x}_{i}^{2}-\sum_{i=1}^{n} \widetilde{y}_{i}^{2}\right)^{2}+4\left(\sum_{i=1}^{n} \widetilde{x}_{i} \widetilde{y}_{i}\right)^{2}} \\
\tan \theta & =\frac{A+B}{C}
\end{aligned}
$$

where $x_{i}$ and $y_{i}$ are the coordinates of the point, $\bar{X}$ and $\bar{Y}$ are the arithmetic mean center, $S D E x$ and SDEy are the ellipse center; $\tan \theta$ is the ellipse azimuth. The long axis $\left(\sigma_{x}\right)$ and short axis $\left(\sigma_{y}\right)$ are calculated as follows:

$$
\begin{gathered}
\sigma_{x}=\sqrt{2} \sqrt{\frac{\sum_{i=1}^{n}\left(\widetilde{x}_{i} \cos \theta-\widetilde{y}_{i} \sin \theta\right)^{2}}{n}} \\
\sigma_{y}=\sqrt{2} \sqrt{\frac{\sum_{i=1}^{n}\left(\widetilde{x}_{i} \sin \theta+\widetilde{y}_{i} \cos \theta\right)^{2}}{n}}
\end{gathered}
$$

The standard deviation ellipse can be used to quantitatively describe the degree of dispersion and directionality of the spatial distribution of time-honored restaurants. The long axis of the ellipse represents the main direction of the distribution of time-honored brands, and the short axis represents the minimum diffused direction of the brands.

\subsubsection{Buffer Analysis}

Buffer analysis is a spatial analysis method used to identify the impact of surrounding features on geographic entities [32]. The buffer zone refers to the spatial influence or service range of a certain geographic entity. This article uses buffer analysis to establish a certain range of buffer areas around the city's main roads to determine whether traffic has an impact on the spatial distribution of Chinese Time-honored Brands.

\section{Results}

\subsection{Basic Information of CTCBs}

\subsubsection{Brand and Quantity}

The origins of the brands studied in this paper can be divided into the five provinces in northwest China and the places outside the five provinces. There are 29 brands from the northwest region, with a total of 392 outlets, accounting for about $32.32 \%$ of all outlets distributed in the five northwest provinces and there are 45 brands originating from other provinces distributed in the five provinces, with a total of 821 outlets, covering 23 provinces (Figure 1). As a whole, there are 74 brands, 1213 outlets in total. Among them, the timehonored brands originating from Shaanxi, Guizhou, Sichuan, and Beijing rank among the top four in the distribution of the five provinces, accounting for $19.46 \%, 18.05 \%, 13.85 \%$, 
and $10.22 \%$ of the total number respectively. From the distribution of old brands in the five provinces, there are 654 points in Shaanxi, accounting for 53.92\% of the number of all outlets, accounting for the largest proportion; 319 stores are distributed in Gansu, accounting for $26.30 \%$; there are 98 in Xinjiang and 93 outlets in Ningxia, accounting for $8.08 \%$ and $7.67 \%$ respectively; Qinghai has the least, with only 49 , accounting for $4.04 \%$ (Figure 1).

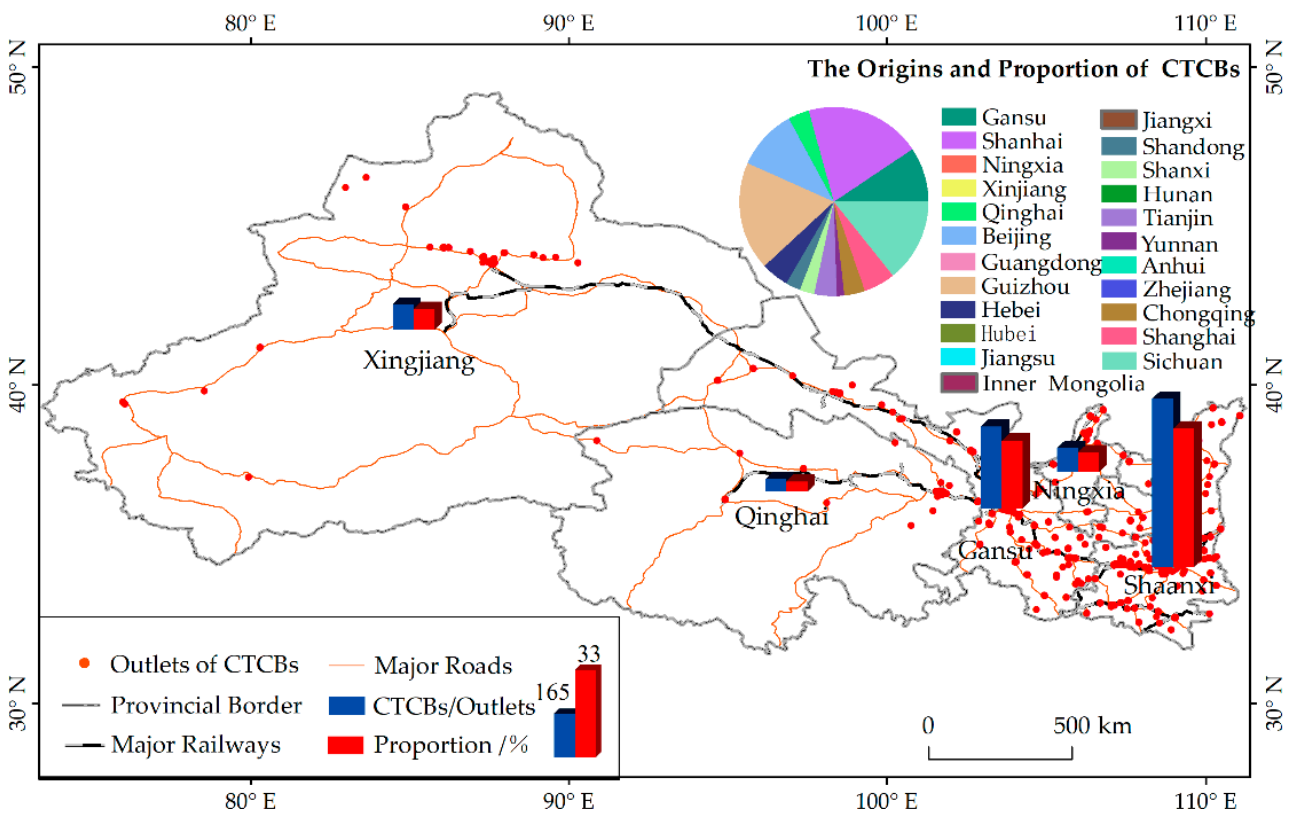

Figure 1. The existing outlets and proportion of Chinese Time-honored Catering Brands (CTCBs) in the five northwest provinces, the source provinces, and their proportion and spatial distribution.

\subsubsection{Basic Distribution in the Five Northwest Provinces}

The spatial distribution of CTCBs in the five northwestern provinces is shown in Figure 1. It can be seen that the sales outlets of CTCBs are found almost everywhere in the study area, but there are more brands concentrated in the east, especially in Shaanxi, while compared with the east, the distribution of time-honored brands is sparser in the west.

\subsection{Spatial Distribution Characteristics}

\subsubsection{Calculation of Regional Development Intensity}

The calculation of the location entropy of CTCBs in the five northwestern provinces is based on the default premise that the capacity of the number of brands in each province is fixed, otherwise, the spatial layout of them in different provinces would not be comparable [33] (Table 1). It can be seen that the development intensity of the time-honored catering industry is strong in the place of origin. There are 23 provinces (municipalities directly under the Central Government) with corresponding catering businesses distributed in the five provinces. Except for the time-honored restaurants, which come from the northwestern part of the country and occupy about 32.32\%, the time-honored restaurants from neighboring regions of five provinces (Tibet, Inner Mongolia, Hunan, Shanxi, Sichuan, Hubei, and Chongqing) account for $20.93 \%$. The three provinces of Shanxi, Sichuan, and Chongqing account for the largest proportion, $94.49 \%$ in neighboring areas.

Table 1. Location entropy of time-honored catering brands in the five northwestern provinces.

\begin{tabular}{lccccc}
\hline Province & Gansu & Shaanxi & Ningxia & Qinghai & Xinjiang \\
\hline $\begin{array}{l}\text { Location } \\
\text { Entropy }\end{array}$ & 10.7 & 12.6 & 28.6 & 22.2 & 7.3 \\
\hline
\end{tabular}




\subsubsection{Basic Distribution in The Five Northwest Provinces}

The average nearest distance under observation distribution and the nearest distance under random distribution mode are obtained by applying the method of nearest neighbor analysis (NNA) (Table 2), where $\mathrm{Z}$ is -57.19 , the standard nearest distance index $\mathrm{R}$ is obviously less than 1 , only 0.14 , and the significance level $p$-value is 0.00 , indicating that the distribution of Chinese time-honored restaurants in the five provinces is highly concentrated.

Table 2. Results of nearest neighbor distance.

\begin{tabular}{ccc}
\hline $\begin{array}{c}\text { Average Nearest Distance } \\
\text { under Observation } \\
\text { Distribution }\end{array}$ & $\begin{array}{c}\text { Nearest Distance under } \\
\text { Random Distribution }\end{array}$ & $\begin{array}{c}\text { Standard Nearest Distance } \\
\text { Index R }\end{array}$ \\
\hline 3733.58 & $25,893.86$ & 0.14 \\
\hline
\end{tabular}

\subsubsection{Analysis of Kernel Density and Standard Deviation Elliptic}

From the distribution of the kernel density and standard deviation elliptic of CTCBs in five northwestern provinces (Figure 2), it can be seen that there are significant regional differences in the nuclear density, there is a higher nuclear density in the east, therefore the distribution of CTCBs is concentrated in the east; multiple centers of concentration are distributed in a circle, the most obvious place is $\mathrm{Xi}^{\prime}$ an which situated in Shaanxi province, with the strongest concentration. This is followed by Lanzhou in Gansu province, Xining in Qinghai province, and Yinchuan in Ningxia province. There are few points in the west, which are scattered and mainly concentrated in Urumqi, Xinjiang. The standard deviation ellipse with an azimuth angle of $111.49^{\circ}$ covers $95 \%$ of the time-honored outlets. Its long semi-axis of the standard deviation ellipse is $19,146.55 \mathrm{~m}$ and the short semi-axis is only $4143.54 \mathrm{~m}$. The oblateness of the ellipse is large and the brands are obviously distributed in the northwest-southeast direction. It means that the spatial distribution of the CTCBs in the five northwestern provinces is concentrated and directional.

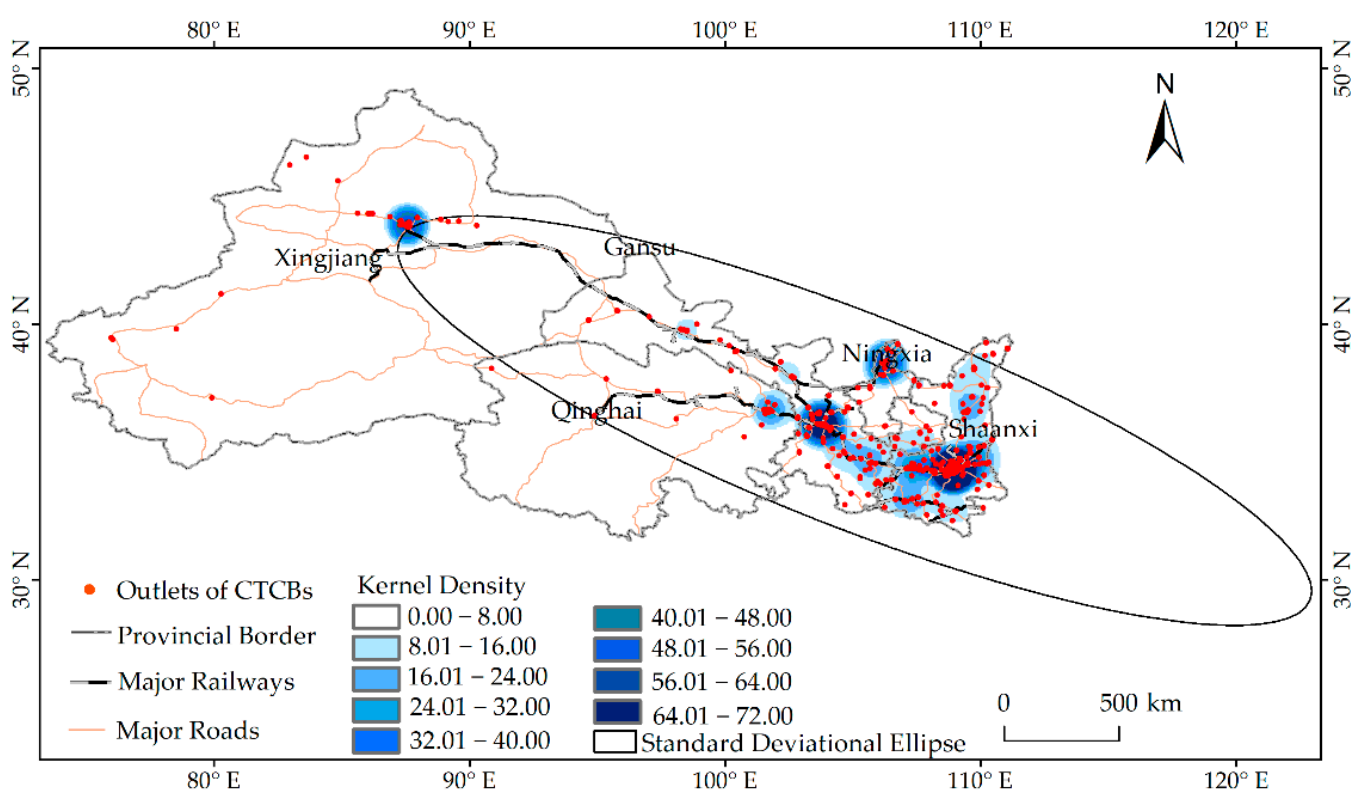

Figure 2. Distribution of kernel density and standard deviational ellipse of CTCBs in the five northwest provinces.

\subsubsection{Spatial Distribution of CTCBs with Different Flavors}

There are a large number of time-honored brands with a wide range of sources. According to the characteristics of Chinese cuisines, there are various cuisines in the 
time-honored catering brands in the five provinces, including Shaanxi cuisine, Beijing cuisine, Sichuan cuisine, Hubei cuisine, Tianjin cuisine, Shandong cuisine, Jiangsu cuisine, Chongqing cuisine, Zhejiang cuisine, Northeast cuisine, Anhui cuisine, Shanghai cuisine, Muslim cuisine, etc. In order to make it more convenient for us to organize it, this article classifies all the flavors of CTCBs into three categories: Northwest local flavors (excluding Muslim foods), Muslim flavors, and other flavors [34,35] (Figure 3). Among them, there are 359 outlets with local flavors in the five provinces, accounting for $29.60 \%$ of all existing outlets of CTCBs. Their agglomeration is higher in the east and gradually decreases from east to west, which is similar to all of the CTCBs which we have analyzed above. They are mainly distributed in Urumqi and Xinjiang in the west, and their diffusion scope and agglomeration degree are far less than those in the east. In general, Northwest local CTCBs are located in all major Northwest provinces, with small concentrations in their provincial seat. The proportion of Muslim flavors among all the outlets is relatively small, 126 in total, accounting for about $10.39 \%$. Northwest China is a concentrated area of ethnic minorities, inhabited by a large number of Hui people, which has affected the types of old brands in Northwest China in their long history, making the distribution of Muslim old brands spotty and concentrated. The number of other flavors is the largest, 731 in total, accounting for $60.01 \%$ of all time-honored brands, its distribution characteristics are similar to the types of time-honored brands, but the distribution scope is wide and the aggregation is weakened.

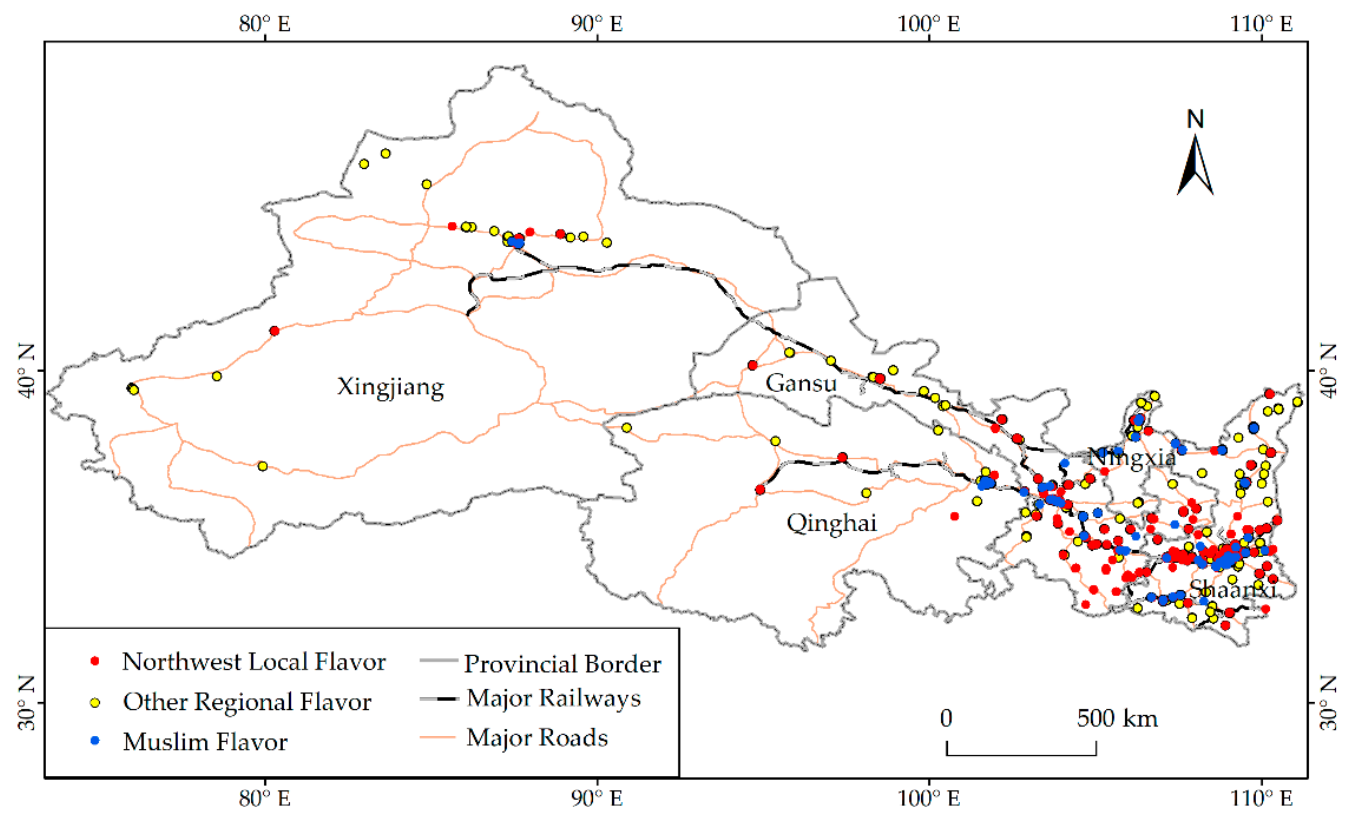

Figure 3. Spatial distribution of CTCBs with different flavors in the five northwest provinces.

Other flavors of CTCBs occupy the largest market in the northwest region, while local time-honored brands are only half of the other flavors of time-honored brands in quantity. In addition, the number of time-honored brands with high distribution density is small, such as Dukang, Xifeng Liquor, etc. It shows that the development advantages of local time-honored brands are inferior to other types, and there is still much room for development.

\subsubsection{Spatial Distribution of CTCBs with Different Consumption Levels}

The overall consumption level of the research objects in this paper is between 1 and 1000 yuan, so the time-honored brands are divided into three grades according to the per capita consumption level [36]: 0-50 yuan is classified as low-grade (including 50 yuan), mainly snacks, and the representative brands are Lanzhou Ramen, Tianjin Goubuli and Tsingtao Beer; $50-100$ yuan (including 100 yuan) is considered to be medium-grade, mainly for dinners, such as $\mathrm{Xi}^{\prime}$ an Restaurant, Hongbinlou, and Shaguoju restaurant; high-grade 
catering consumption is above 100 yuan, mainly wine and tea, such as Chinese Moutai, Luzhou Laojiao and Xiaguan Tuocha. There are 403 low-grade time-honored brands, 619 medium-grade time-honored brands, and 191 high-grade time-honored brands. According to ArcGIS10.4 standard deviation ellipse analysis (Table 3), it is obvious that the long and short semi-axes and azimuth angles of the three grades of time-honored restaurants have little difference. The elliptic long semi-axis of the low-grade brands are longer and shorter than those of the other two grades, $105.62 \mathrm{~km}$, and $35.94 \mathrm{~km}$, respectively, which means that the spatial distribution of the low-grade brands is more concentrated than those of the other two grades, but in short, there is little difference. However, the difference between the long semi-axis and short semi-axis values of all grades is large, and the agglomeration is obvious. All of them extend in the northwest-southeast direction (Figure 4).

Table 3. Standard deviation ellipse analysis results of different grades of CTCBs in the five northwestern provinces.

\begin{tabular}{cccc}
\hline Grade & Long Semi-Axes/km & Short Semi-Axes/km & ${\text { Azimuth } \text { Anglel }^{\circ}}^{\text {Low-grade }}$ \\
Medium-grade & 105.62 & 35.94 & 112.22 \\
High-grade & 104.76 & 36.40 & 110.65 \\
\hline
\end{tabular}

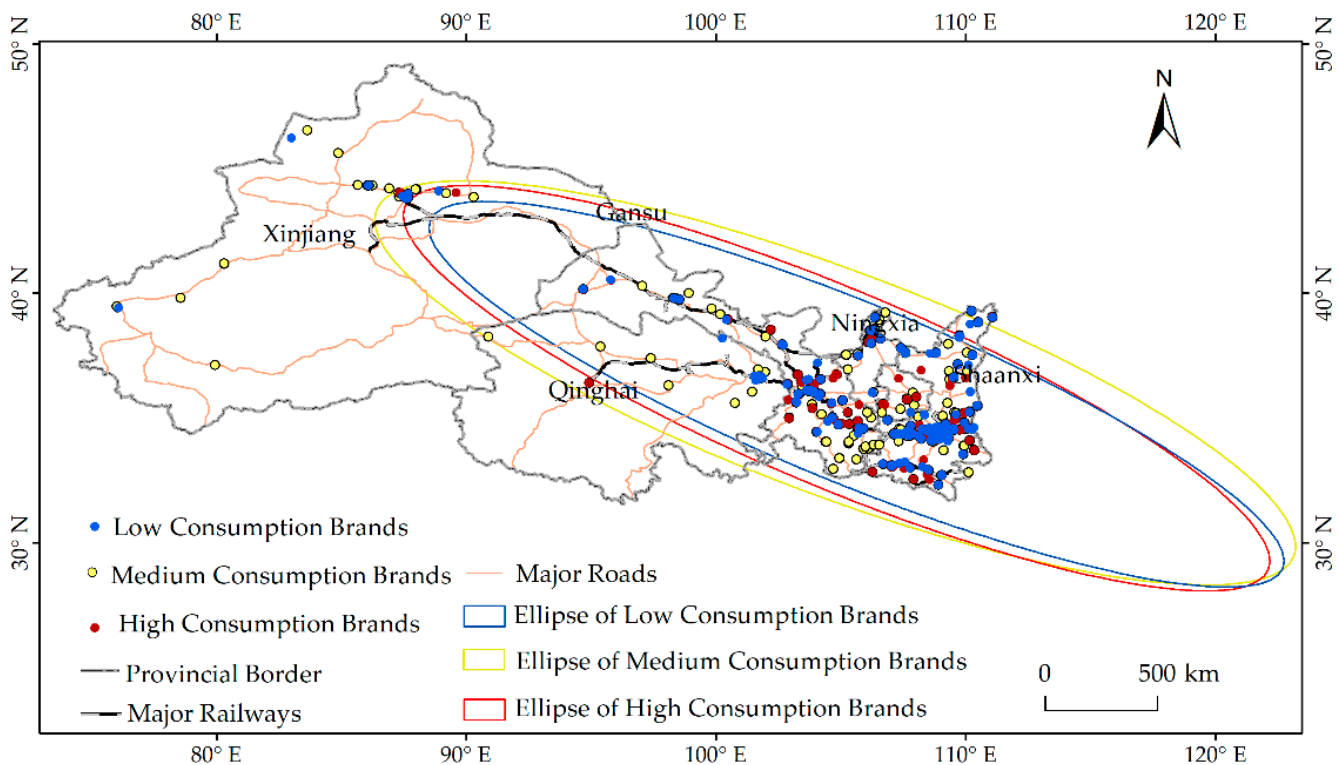

Figure 4. Distribution of standard deviational ellipse of different grades of CTCBs in the five northwest provinces.

The kernel density analysis results of the three grades of time-honored outlets (Figure $5 \mathrm{a}-\mathrm{c}$ ) show that the number of low-grade outlets accounts for $33.22 \%$ of all old outlets, covering 37 brands, including Tianjin Goubuli Steamed Bun, Shandong Tsingtao Beer, Lanzhou Mazilu Beef Noodle, etc. Middle-grade time-honored stores account for the highest proportion, accounting for $51.03 \%$ of all stores, covering 29 brands, such as the famous Lanzhou Jing-yang Tower, $\mathrm{Xi}^{\prime}$ an Casserole, Suzhou Song-he Tower, etc. High-grade time-honored brands account for $15.75 \%$ of all time-honored brands, accounting for the lowest proportion, covering seven brands, including Old Cellar in Luzhou, Maotai Liquor, etc. In addition, the nuclear density value is far less than that of middle and low-grade brands. There are two obvious assembled centers for all three grades of CTCBs, they are $\mathrm{Xi}^{\prime}$ an and Lanzhou, and the sub-level is located in the part of Shaanxi and Gansu Province and Urumqi in Xinjiang, while the other places are more 
scattered. The numbers of shop-fronts of a lower grade are more than those of a higher grade, and the diffusion range is also slightly wider.

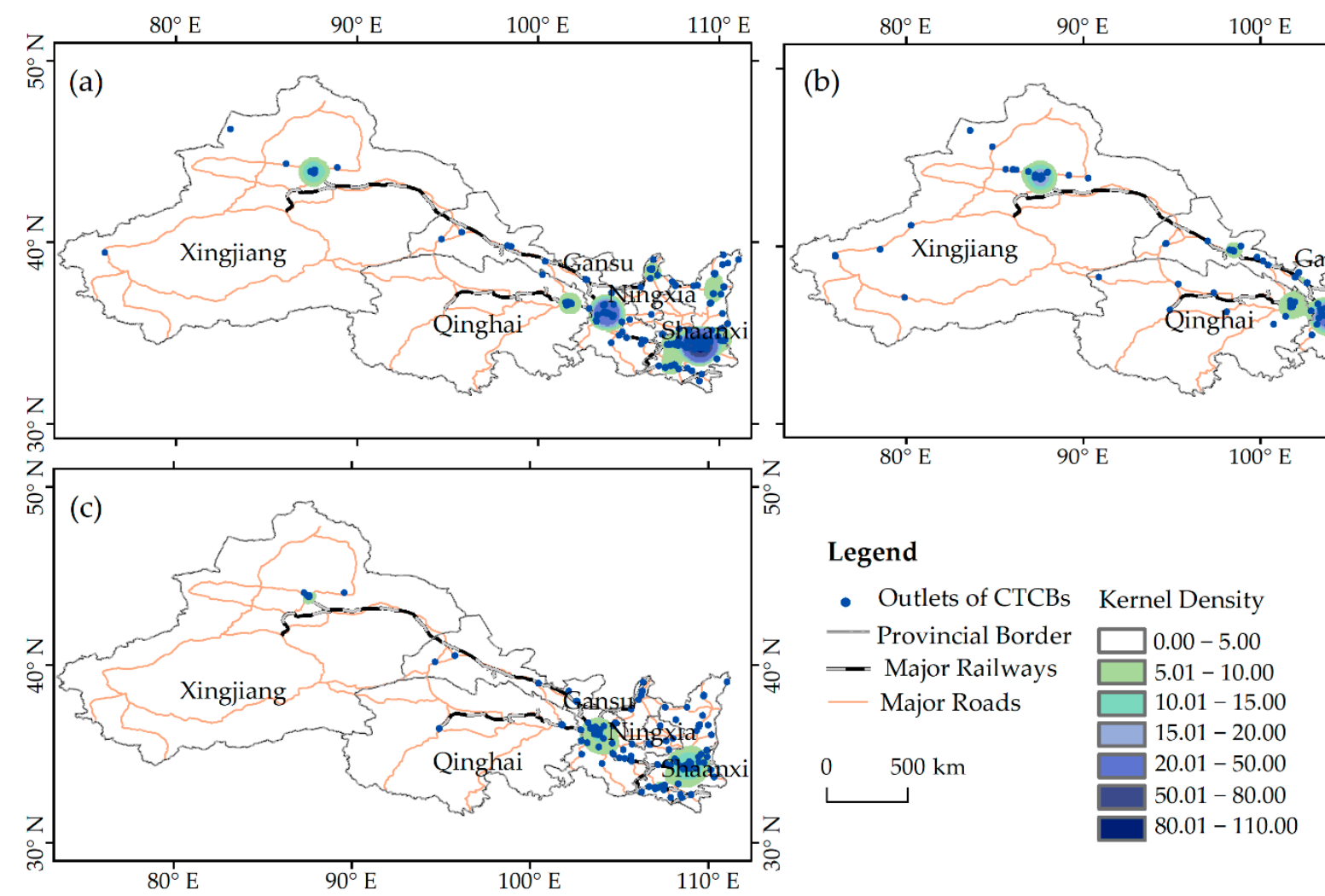

Figure 5. Core density diagram of low-grade (a), middle-grade (b), and high-grade (c) time-honored brands.

\subsection{Analysis of Influencing Factors}

\subsubsection{Traditional Culture}

The CTCB in Northwest China is a business card for tourists to recognize the city. It is a microcosm of the traditional culture of the northwest region and a special historical and cultural landscape with a profound traditional cultural foundation. Especially Lanzhou beef noodles, Xi'an Chinese hamburger, Luzhu highland barley wine, and so on, their existence is not only food but also a symbol of culture [37]. Every time-honored brand is a product of traditional culture and has strong regional characteristics. Original culture is the main influencing factor of the CTCBs' spatial distribution which makes the catering brands form unique tastes. Since ancient times, the northwest region has gathered and lived with a large number of ethnic minorities, who have different religious beliefs and engage in unique production and economic activities, the result is that the development of their diet is closely related to the traditional culture of local cities.

A city's historical and traditional culture determines the eating habits of the people in the area. With the influence of the long-term historical and traditional culture of the city, lots of CTCBs with the characteristics of this city have been formed. Even with the gradual opening up of economy and culture, local catering cultures blend with each other, the time-honored brands still have the tastes that only belong to their origin [38].

\subsubsection{Traffic Conditions}

In order to clearly reflect the impact of traffic on the layout of CTCBs, we use ArcGIS 10.4 to establish $10 \mathrm{~km}, 20 \mathrm{~km}$, and $30 \mathrm{~km}$ buffer zones for the major railways and roads of the five northwestern provinces (Figure 6). There are 1043 outlets of CTCBs within $10 \mathrm{~km}$ of the major highways in the five provinces, accounting for $85.60 \%$ of all points, 1094 outlets within $20 \mathrm{~km}$, accounting for $90.20 \%$, and 1152 outlets within $30 \mathrm{~km}$, accounting for $95.00 \%$, 
only $5 \%$ of which are not included. There are 766 outlets within $10 \mathrm{~km}$ of the major roads in the five northwestern provinces, accounting for $61.15 \%$ of all points, 923 outlets within $20 \mathrm{~km}$, accounting for $75.09 \%$, and 980 points within $30 \mathrm{~km}$, accounting for $80.79 \%$. It can be seen that the spatial layout of CTCBs is obviously affected by the traffic conditions. Convenient transportation has promoted the development of CTCBs along the route: the continuous improvement of traffic conditions and the increasing coverage of the traffic network have attracted people from different places and brought momentum to the development of time-honored brands. Most of the areas with developed transportation networks are populated areas [39]. For example, there are plenty of tourists around bus stations and railway stations, which have good business conditions. Road traffic network pattern promotes the distribution pattern of "core-edge" of catering facilities and the layout of static transportation facilities is also a key factor to improve the transportation convenience of catering facilities.

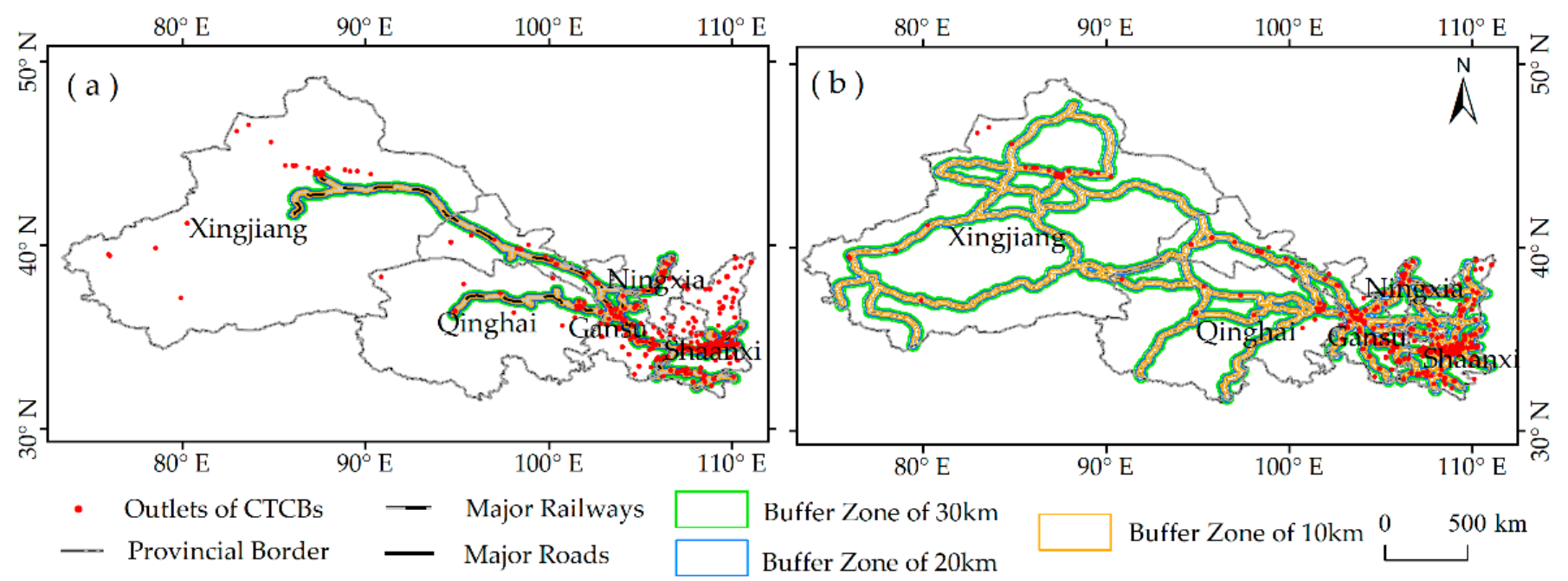

Figure 6. Influence of main roads (a) and railways (b) on the distribution of time-honored brands in the five northwestern provinces.

\subsubsection{Distribution of the Population}

The regional population distribution determines the spatial layout and development intensity of the catering industry, which in turn strongly affects the coupling of the types, quantity, and spatial distribution of CTCBs. We overlay the resident population distribution map of the five northwestern provinces in 2018 with the distribution map of time-honored brands (Figure 7). It is obvious that the density of the two has a high spatial consistency. Pearson correlation analysis of the two elements shows that the correlation coefficient is $0.815(p<0.01)$, which indicates there is a significant correlation between them. The density of population will directly affect the number and consumption power of consumers in the region, make the number and types of brands increase to meet the needs of different consumers, and promote the prosperity of CTCBs.

\subsubsection{Economic Development Level}

In order to reveal the relationship between the spatial distribution of CTCBs and the level of regional economic development, we overlay the GDP distribution map of the five northwestern provinces in 2018 with the distribution map of time-honored brands (Figure 8 ), which clearly shows that time-honored brands are mostly concentrated in cities with higher GDP. On the contrary, the number of time-honored brands in cities with lower GDP is smaller and the density is lower. The Pearson correlation coefficient of the two is $0.914(p<0.01)$ and the correlation between the two elements is significant. Time-honored brands are generally highly agglomerated in provincial capitals, such as $\mathrm{Xi}^{\prime}$ an, Lanzhou, Urumqi, etc., which are closely related to their GDP. The improvement of the regional 
economy will significantly stimulate people's consumption and make people pursue more diversified, high-service, and connotative consumption, which will drive the expansion in space and increase the number of time-honored stores.

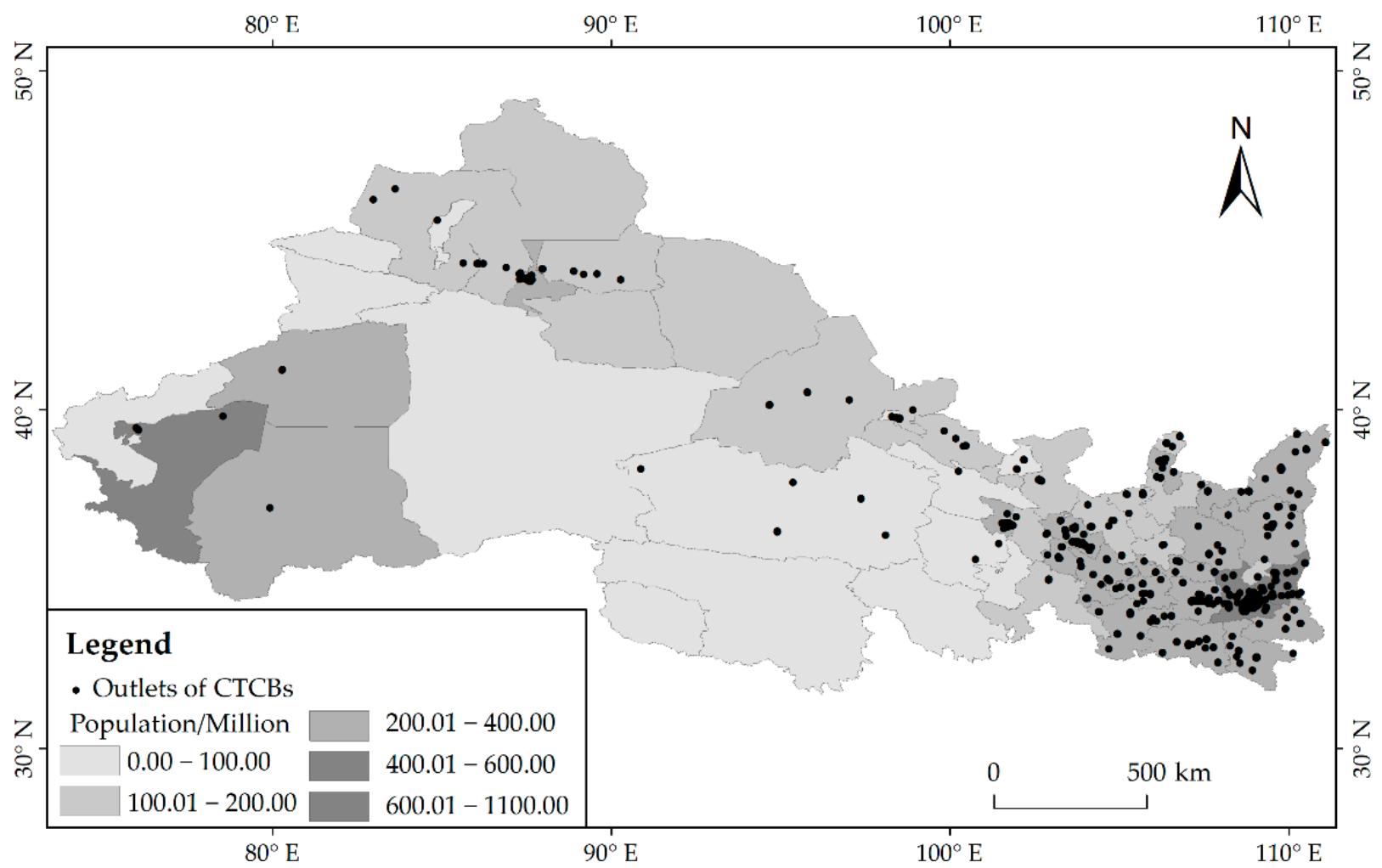

Figure 7. Superimposed diagram of time-limited restaurants in five northwest Provinces and population.

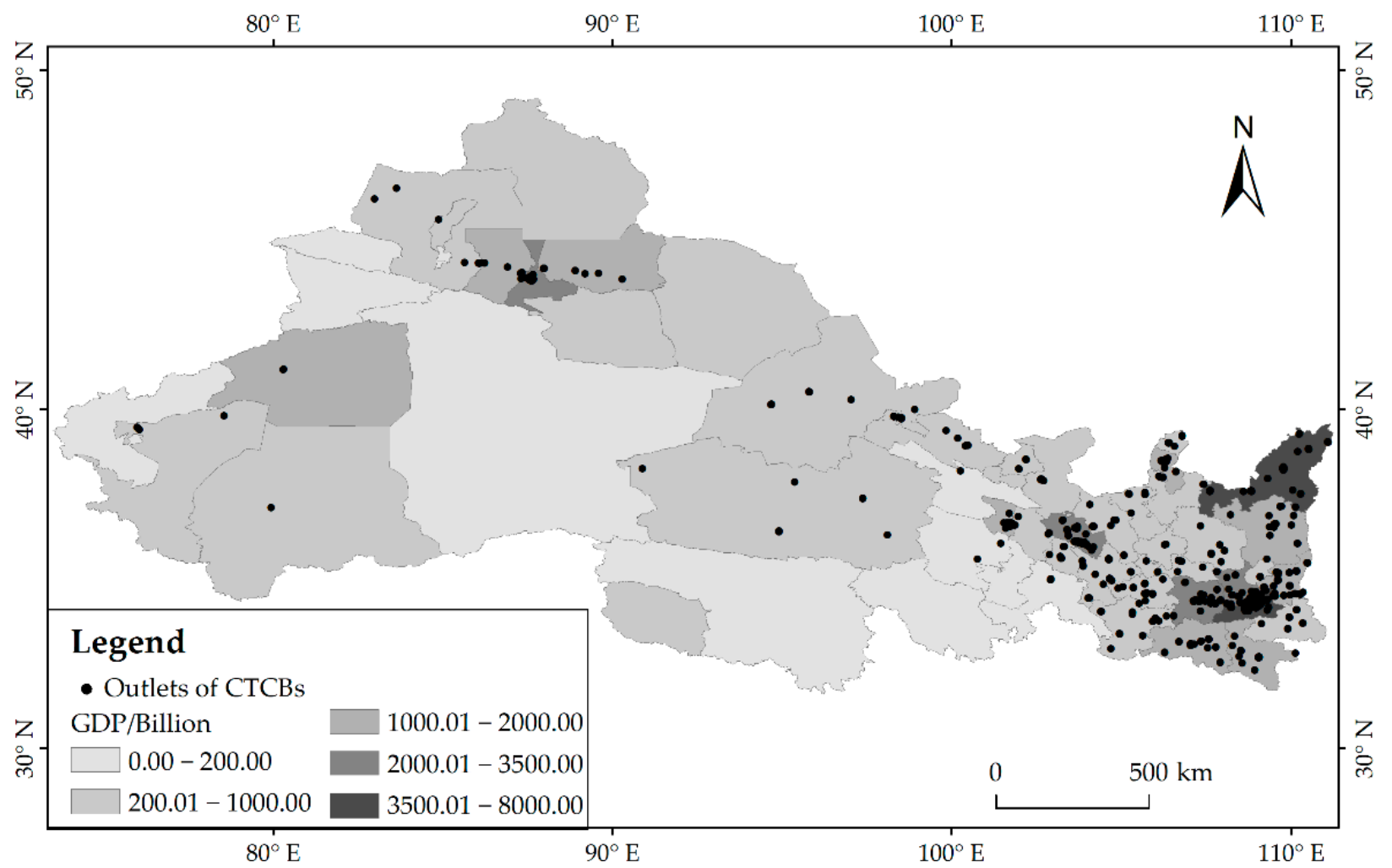

Figure 8. Superimposed diagram of time-honored restaurants in five northwest provinces and urban GDP. 


\subsubsection{Urban Development Pattern}

The overall planning of the city determines the pattern of urban development, which in turn affects the spatial layout of the urban catering industry [40]. The urban agglomerations in the northwest of China are in the process of continuous construction and development. The development of each province is still focused on the provincial capital city, and the development of the northwest cities presents obvious polarization. Therefore, the spatial distribution of CTCBs is also centered on provincial capitals. In addition, the intensity of central functions in different cities varies, the central functions of cities in Shaanxi Province are generally stronger [41], $\mathrm{Xi}^{\prime}$ an is the main representation. So the time-honored brands are concentrated in Shaanxi Province mostly. At present, with the changes of urban planning in the northwestern regions, the single-core model of the urban layout is gradually evolving to the multi-center model. The functions of cities are becoming more and more perfect, making the distribution of CTCBs change accordingly. With the establishment of urban agglomeration, the accessibility and the links among regions are strengthened, and the functions of many cities are undergoing a transformation from traditional industrial cities to regional modern central cities. The area of the central city continues to expand in the direction of multi-dimensionality, which promotes the agglomeration of old brands in the urban area.

\section{Discussion}

In this paper, we take the five northwestern provinces as the study area, and apply statistical analysis, kernel density analysis, standard deviational ellipse, and buffer analysis to analyze the spatial distribution characteristics and influencing factors of CTCBs. The results show that the existing CTCBs in the five northwestern provinces have a certain number and spatial scale, with various tastes and grades. However, their spatial layout is uneven and their coverage is not comprehensive. The spatial distribution of CTCBs is obviously different between east and west. Moreover, compared with CTCBs from other regions, the local CTCBs in the five northwestern provinces are not dominant in quantity, and their competitiveness is weak, which is not conducive to the sustainable development of time-honored brands in the region. This spatial distribution characteristic is the result of the combined effect of multiple factors. Traditional culture has played a more decisive role. It is the basis of the formation of CTCBs, determines the types and quantities of time-honored brands, and is the direct cause of the current spatial pattern. Regional traffic conditions make CTCBs densely distributed in convenient transportation areas. The regional population and economic level directly affect the number and grade distribution of time-honored brands. The urban development pattern further affects the spatial distribution characteristics of the time-honored catering industry and restricts the future development trend of the CTCBs.

It can be seen from the results that the CTCBs in the five northwestern provinces, whether in terms of quantity or spatial distribution, still have a lot of room for improvement in the face of sustainable development goals. In recent years, with the popularity of multiculturalism and new culture, it has not only brought certain opportunities but also shock to the sustainable development of CTCBs [42]. Some time-honored brands have seized the opportunity to go abroad under the background of globalization. For example, Moutai, as a national liquor, has received rave reviews from scientists and consumers from all over the world [43]. However, most CTCBs have been restricted due to fierce competition. Therefore, based on the spatial differentiation pattern, type structure characteristics, and current sustainable development dilemmas of CTCBs in the northwest of China, this study puts forward the following development suggestions:

(1) Giving policy support. The government provides policy support for certain brand protection of time-honored brands, which can prolong the life of time-honored brands and provide a guarantee for their sustainable development. For example, the project of "Revitalization of Time-honored Brands" implemented by the Ministry of Commerce in 2006 has greatly improved the social status of time-honored brands [44] and 
the "Belt and Road" initiative has made the international cooperation of Chinese enterprises more frequent and in-depth [45]. Many time-honored enterprises actively explore overseas markets, which has brought unprecedented sustainable development opportunities to the inland areas, especially in the five northwestern provinces.

(2) Carrying out sustainability-oriented innovation. Forêt pointed out that the only path to sustainable growth in the domestic and overseas is sustainability-oriented innovation, rather than relying on longevity [46]. Therefore, sustainability-oriented innovation should be focused on the development of CTCBs. This kind of innovation is all-around and multi-dimensional. It is not only necessary to innovate development concepts, change traditional development thinking, break the inherent pattern, and enhance the market vitality of time-honored brands at multiple levels and angles; but also necessary to keep up with the new era of mobile Internet development, create a new sustainability-oriented label and skillfully use big data analysis, experience rating [13], fan interaction, and other methods to create a new and sustainable sales model and avoid falling behind the times.

(3) Improving the sustainability of quality and reputation. Customer word-of-mouth plays a key role in brand inheritance and reputation [47,48], especially the CTCBs, its sustainable development mainly depends on the sustainable development of customer's word-of-mouth, such as Lanzhou Noodles, whose strong taste is familiar to people. Therefore, the sustainable development of CTCBs in the area not only relies on signs but also requires the inheritance of culture and craftsmanship to ensure its quality and increase customer's reputation.

Since this paper takes the existing CTCBs in the five northwestern provinces as the research object, the obtained conclusions are based on the findings of the five provinces, and the CTCBs have strong regional characteristics, so the results obtained in this article have certain limitations. Spatial distribution characteristics and influencing factors of CTCBs in other regions will be studied in future research.

\section{Conclusions}

The time-honored catering enterprises in China are important products of the Chinese society, economy, and culture, and their long history of development and management is an indispensable part of the development of Chinese historical civilization. This paper analyzes the spatial distribution pattern and influencing factors of CTCBs in the five northwestern provinces of China and draws the following conclusions:

(1) The intensity of CTCB's spatial development in the five northwestern provinces is strong in their own birthplaces. In addition, the outlets of CTCBs are unevenly distributed in the northwest-southeast direction, they are highly concentrated in the provincial capitals and tend to be scattered in the periphery of the concentrated areas. Some areas with high concentration discretely exist in the form of patches, and the agglomeration gradually increases from west to east.

(2) The number of time-honored brands in different grades and tastes varies greatly, but their spatial distribution characteristics are similar, all of which are distributed unevenly in the northwest-southeast direction.

(3) The spatial distribution pattern of time-honored brands is influenced by many factors, such as history, traditional culture, traffic conditions, regional population and economic development level, urban development pattern, etc. These factors determine the origins, tastes, types of time-honored brands, and further affect the spatial layout of brands.

Author Contributions: All of the authors contributed to this research. H.Z. (Haiyan Zhang) conceived the study and was responsible for the design and development of the data analysis. H.Z. (Hao Zhang) and M.S. were responsible for data collection and analysis. Z.W. and L.Z. was responsible for data interpretation. H.Z. (Hao Zhang) wrote the first draft of the article. All authors have read and agreed to the published version of the manuscript. 
Funding: This research was funded by the National Natural Science Foundation of China (41801052, 41861013).

Institutional Review Board Statement: Not applicable.

Informed Consent Statement: Not applicable.

Data Availability Statement: Publicly available datasets were analyzed in this study. This data can be found here: The data of the CTCBs' directory was available online from http:/ / zhlzh.mofcom.gov.cn/ (accessed on 30 November 2020). The location data was available online from http:/ / api.map.baidu. com/lbsapi/getpoint/ (accessed on 30 November 2020). The price data for brands was available online from Dianping.com.

Acknowledgments: We greatly thank the colleagues in the Northwest Normal University for their help in fieldwork and data processing. We are grateful to anonymous reviewers and editorial staff for their constructive and helpful suggestions.

Conflicts of Interest: The authors declare no conflict of interest.

\section{References}

1. Tan, X.; Huang, D.Q.; Zhao, X.S. A Study on the Spatial Distribution Pattern of Restaurants in Beijing's Main Urban Area. Tour. Trib. 2016, 31, 75-85.

2. Ministry of Commerce of the People's Republic of China. The Ministry of Commerce and other 16 Departments about Guidance to Promote the Development of Time-honored Brand Innovation. Available online: http://www.mofcom.gov.cn/article/b/d/20 1702/20170202509727.shtml (accessed on 3 February 2017).

3. Ministry of Commerce of the People's Republic of China. Notice of the Ministry of Commerce on the Implementation of the "Project to Revitalize Time-honored Brands". Available online: http://ltfzs.mofcom.gov.cn/article/aw/200604/20060401910767. shtml (accessed on 23 November 2020).

4. Lei, Y.; Xu, P.W. The Classification, Spatial Pattern and Consumer's Network Evaluation of Time-honored Catering Brands in Beijing. Mod. Urb. Res. 2017, 68-75. [CrossRef]

5. Ma, B.B.; Cheng, X.P.; Cheng, F.T.; Cheng, Y.B.; Ding, B.W.P. Spatial differentiation and influencing factors of China time-honored brand. Geo. Res. 2020, 39, 2313-2329. [CrossRef]

6. Wang, Z.; Wang, C.R. The Relationship between R\&D Innovation and Brand Growth of "Time-honored Brand" Enterprises. Manage. Rev. 2020, 12, 156-167. [CrossRef]

7. Schroeder, J.; Borgerson, J.; Wu, Z. A Brand Culture Approach to Chinese Cultural Heritage Brands. J. Brand. Manag. 2015, 22, 261-279. [CrossRef]

8. Liu, Q. Understanding and historical origin of Chinese time-honored brands. Mod. Mark. 2012, 7, 10-11.

9. Soo, P.S.; Heun, K. The Effect of Chinese Time-honored Brand Identity Image on Purchasing Intention. J. North. Asian. Econ. Stu. 2016, 28, 297-325.

10. Henri, W.J.; Bean, J.; Rintamäki, J. Brand community coping. J. Bus. Res. 2017, 94, 128-136. [CrossRef]

11. Ugaglia, A.A.; Cardebat, J.M.; Jiao, L. The French wine industry. Palgr. Hand. Wine. Ind. Econ. 2019, 17-46. [CrossRef]

12. Dressler, M.; Paunovic, I. A Typology of Winery SME Brand Strategies with Implications for Sustainability Communication and Co-Creation. Sustainability 2021, 13, 805. [CrossRef]

13. Dressler, M.; Paunovic, I. The Value of Consistency: Portfolio Labeling Strategies and Impact on Winery Brand Equity. Sustainability 2021, 13, 1400. [CrossRef]

14. Zhang, Y.F. An Analysis of the Current Situation of Beijing Time-honored Catering Enterprises and Study on Their Growth Mechanism. Tour. Trib. 2009, 24, 48-54.

15. Ye, W.P.; Zhao, Q. Innovative Strategies of Marketing Management in Time-honored Enterprises. Econ. Trade. Upd. 2017, 3, 52-54. [CrossRef]

16. Ding, Y.X.; Wang, L.X. Research on the development status, Problems and countermeasures of Traditional time-honored brands in Shanghai. Chin. J. Commer. 2020, 3, 10-13. [CrossRef]

17. Liu, C.; Feng, Y.Q. Spatial distribution characteristics of cross-cultural communication of intangible cultural heritage in Europe-A case study of Shadow Play. World Reg. Stu. 2020, 29, 867-874. [CrossRef]

18. Fang, J.W. The Distribution Characteristics and Clustering Mechanism of Korean Catering Service in Wudaokou District, Beijing. Econ. Geo. 2014, 34, 106-113. [CrossRef]

19. Qin, X.; Zhen, F.; Zhu, S.J.; Xi, G.L. Spatial Pattern of Catering Industry in Nanjing Urban Area Based on the Degree of Public Praise from Internet: A Case Study of Dianping.com. Sci. Geo. Sin. 2014, 34, 810-817. [CrossRef]

20. Zhang, Z.; Myeongcheol, C. The vinegar of China time-honored brand-The success reason analysis of HengShun enterprise. J. Sin. Chin. Stu. 2018, 77, 287-307. [CrossRef]

21. Jin, H.; Miao, Y.T.; Park, S.T. Research on Brand Communication of Chinese Time-honored Brands. J. Ind. Conv. 2018, 16, 9-17. 
22. Guo, J.J.; Kwon, K.J. The Remodeling of Brand Image of the Time-honored Restaurant Brand of Wuhan based on Emotional Design in the Age of Experience Economy. Adv. Soi. Sci. Educ. Hum. Res. 2018, 180, 233-236.

23. Mu, J.K. The Study on Activation Strategy of Time-honored Brand. MATEC Web. Conf. EDP Sci. 2017, 100, 05043. [CrossRef]

24. Zhang, C.Y. Research on Micro-marketing Strategy of China Time-honored Brands. Adv. Eco. Bus. Manag. Res. 2019, 70, 102-106.

25. Jia, Y.Y.; Hu, J.; Liu, D.J.; Liu, Y.R. Spatial distribution of China Time-Honored Brand and the influencing factors. J. Arid. Land. Resour. Environ. 2020, 34, 85-93. [CrossRef]

26. Zhou, A.H.; Zhang, Y.S.; Fu, X.; Zhu, H.Y.; Dong, H.N. Study on the Spatial Distribution of Beijing' s Time-honored Brand Caterings and its Influencing Factors. World Reg. Stu. 2015, 24, 150-158. [CrossRef]

27. Zhang, X.; Zhou, E.; Zhang, X.H.; Wang, S.H. Spatial distribution and clustering of commercial network in Beijing during 2004-2008. Prog. Geo. 2013, 32, 1207-1215. [CrossRef]

28. Zhou, J.; Cheng, M.; Wang, L. Research on the Agglomeration level of Financial Industry in Shaanxi Province based on location entropy index. Stat. Decis. 2014, 24, 176-179. [CrossRef]

29. Cai, X.J.; Wu, Z.F.; Chen, J. Analysis of road network pattern and landscape fragmentation based on kernel density estimation. Chin. J. Ecol. 2012, 31, 158-164. [CrossRef]

30. Yang, R.; Liu, Y.S.; Long, H.L.; Wang, Y.; Zhang, Y.J. Spatial Distribution Characteristics and Optimized Reconstructing Analysis of Rural Settlement in China. Sci. Geo. Sin. 2016, 36, 170-179. [CrossRef]

31. Li, H.; Zhang, Z.B.; Wang, W.j. Spatial Distribution and Patterns and Influential Factors of Large and Medium Supermarkets in Lanzhou. Econ. Geo. 2016, 36, 85-93.

32. Tang, G.A.; Liu, X.J.; Run, G.N.; Sheng, Y.H.; Wang, C.; Zhang, T. Geographic Information System Tutorial, 1st ed.; HEP: Beijing, China, 2007; pp. 217-219.

33. Wang, S.; Cheng, Z.G.; Huang, F.F. Spatial Pattern and Location Choice of Chain Supermarkets in Guangzhou. Econ. Geo. 2015, 35, 85-93. [CrossRef]

34. Wang, S.J.; Hao, F.L.; Jiang, L.L. Locations and their determinants of large-scale commercial sites in Changchun. China J. Geo. Sci. 2015, 70, 893-905. [CrossRef]

35. Chen, C.K. The Culture of Chinese Diet: Regional Differentiation and Developing Trends. J. Geo. Sci. 1994, 49, $226-235$.

36. Wang, Y.Z.; Zeng, G. Coupling Development of Cultural Industry and Tourism Industry in Northwest China. Econ. Geo. 2020, 40, 234-240. [CrossRef]

37. Wang, S.M.; He, Z.L.; Guo, Y.Z. Spatial Differentiation and Influencing Factors of Rural Tourism Model Villages in the Five Northwest Provinces of the Silk Road Economic Belt. Econ. Geo. 2019, 39, 199-206. [CrossRef]

38. Ma, G.Q.; Wang, H.L.; Xu, Q. The Symbiotic Development of Tourism Economy and Transportation Industry in Northwest China. Econ. Geo. 2019, 39, 223-230. [CrossRef]

39. Hou, J.; Zhang, M.Q. Study on the Influence of Functional Division of Urban Agglomerations on Regional Coordinated Development-Taking Beijing-Tianjin-Hebei Urban Agglomeration as an Example. Economics 2020, 6, 77-86. [CrossRef]

40. Deng, S.W. Review on Studies of Urban Commercial Network Layout in China. Hum. Geo. 1999, 14, 36-39. [CrossRef]

41. Wang, J. Virtual Community, Online Word-of-Mouth and Consumers' Behavior. Commer. Res. 2011, 12, 71-76. [CrossRef]

42. Li, J.F. Application of Modern Hospitality Management Practices in China's Time-Honored Restaurants; Dissertations; University of Nevada: Las Vegas, NV, USA, 2010.

43. Liu, Y.R. Cultural Analysis and Development Strategy of Kweichow Moutai Liquor. Mod. Food. 2021, 2, 76-78. [CrossRef]

44. Lin, H. Revitalizing Time-honored Brands and Promoting Chinese National Brands-The Ministry of Commerce launched the project of revitalizing time-honored brands. Chin. Food. 2007, 4-5. [CrossRef]

45. Andornino, G. The Belt and Road Initiative in China's Emerging Grand Strategy of Connective Leadership. Chin World Econ. 2017, 25, 4-22. [CrossRef]

46. Forêt, P.; Mazzalovo, G. The long march of the Chinese luxury industry towards globalization: Questioning the relevance of the "China time-honored brand" label. Luxury 2015, 1, 133-153. [CrossRef]

47. He, J.X. Transference or severance: An exploratory study on brand relationship quality of China's time-honored brands based on intergenerational influence. Front. Bus. Res. China 2008, 2, 518-552. [CrossRef]

48. Zhang, S.N.; Li, Y.Q.; Liu, C.; Ruan, W.Q. A study on China's time-honored catering brands: Achieving new inheritance of traditional brands. J. Ret. Cons. Serv. 2021, 58. [CrossRef] 